\title{
On New Knot Tables
}

\author{
Ceren Sultan ELMALI ${ }^{*}$, Tamer UĞUR ${ }^{2}$, Tuğçe KUNDURACI ${ }^{3}$ \\ ${ }^{1}$ Department of Mathematics, Faculty of Science, Erzurum Technical University, 25100 Erzurum-TURKEY \\ ${ }^{2}$ Department of Mathematics, Faculty of Science, Atatürk University, 25200 Erzurum-TURKEY \\ ${ }^{3}$ Graduate School of Natural and Applied Science, Erzurum Technical University, 25100 Erzurum-TURKEY
}

\begin{abstract}
The quasi-pseudo metrics on the vertices of a digraph induces a unique bitopology. In this work, we obtained that a bitopology is associated with any knot $k_{m}$, where $k$ is crossing points of knot and $m=1,2$ by using quasi-pseudo metrics on the vertices of a digraph which is called knot digraph by us, when we consider relation between the knot and its graph (knot digraph notation). Also some topological properties of bitopology obtained by using quasipseudo metric and associated with the knot digraphs are investigated.
\end{abstract}

\section{Introduction}

The mathematical notion of bitopological space $\left(X, \tau_{1}, \tau_{2}\right)$, namely, a set $X$ equipped with two arbitrary topologies $\tau_{1}, \tau_{2}$. For the first time, J.C.Kelley formulated this space [4]. Girija and Plakkat showed that bitopology is get from a digraph which has orientation by using quasi-pseudo metric entitled with 'Bitopological space associated with digraphs' [3] in 2013. A quasi pseudo metric on nonempty set $X$ is a nonnegative real valued function $\rho$ on $X \times X$ such that $\rho(x, x)=0, \forall x \in X$ and $\rho(x, z) \leq \rho(x, y)+\rho(y, z), \forall x, y, z \in X$ associated with $\rho$ there is another quasi pseudometric $q$ such that $\rho(x, y)=q(y, x)$.In this study the authors is called digraph if the vertex of graph is reachable from edge of it. Let be $V$ the vertex set of a digraph. The function $p: V \times V \rightarrow \mathbb{R}$ and $q: V \times V \rightarrow \mathbb{R}$ defined by

$$
p(x, y)=\left\{\begin{array}{lr}
0, & \text { If } x \text { is reachable from } y \\
1, & \text { otherwise }
\end{array}, q(x, y)=\left\{\begin{array}{lr}
0, & \text { If y is reachable from } x \\
1, & \text { otherwise }
\end{array}\right.\right.
$$

respectively. These functions are quasi-pseudo metrics on $V$ and induces a unique bitopology on $V$ with

$S_{p}(x, \varepsilon)=\{y: p(x, y)<\varepsilon\}$ and $S_{q}(x, \varepsilon)=\{y: q(x, y)<\varepsilon\}$ as a basis. All open $\rho$-balls collection forms a base for a topology on $X$. We show this topology with $\tau_{1}$. Similarly all open $q$-balls collection forms a base for a topology on $X$. We show this topology with $\tau_{2}$. So, we get the bitopology $\left(X, \tau_{1}, \tau_{2}\right)$. A bitopological space $\left(X, \tau_{1}, \tau_{2}\right)$ is a set $X$ with two topologies, $\tau_{1}$ and $\tau_{2}$, on it.

We have seen that this method can be applied for the oriented knot graph which is obtained form regular diagram of a knot. We can get a bitopology matched with any knot by using knot digraph when we consider relation between the knot and its graph.

In this way, our purpose contributes the problem of classification the most important problem of mathematic by using bitopology. We plan knot table of prime knot, which has at least 11 crossing by using this method. In our day, there isn't any table of all prime knots. So, we aim to contribute knot table of all prime knot in future period.

\section{Knot and Knot Graphs}

A knot is a simple closed polygonal curve in $R^{3}$ [1]. A knot projection is called a regular projection if no three points on the knot project to the same point, and no vertex projects to the same point as any other point on the knot. It divides the plane into several regions. Each of this regions is homeomorphic to an open disk. It is useful tool for separating regions (domains) into two classes, white-black or shaded-unshaded or shadow-unshadow regions, in the study of the knot theory.

A regular projections of knot $K$ divides plane into $n+2$ regions where $\mathrm{n}$ is the number of double point $A\left(A_{1}, A_{2}\right.$, $\left.\ldots, A_{n}\right)$. The Figure 1 show that the number of double point of Trefoil knot is 3 . For this reason the number of regions is 5 for Trefoil knot.

\footnotetext{
${ }^{*}$ Corresponding Author: ceren.elmali@erzurum.edu.tr
} 


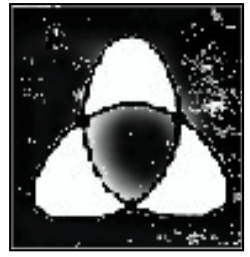

Figure 1

Starting with the outer most region, the regions can be colored black or white where neighboring regions are never this same color. White and black regions are labelled, in order of, such that $\alpha=\left\{\alpha_{1}, \alpha_{2}, \ldots, \alpha_{k}\right\}$ and $\beta=\left\{\beta_{1}, \beta_{2}, \ldots, \beta_{l}\right\}$. The point at infinity is contained in a region of black. $P_{i} \in \alpha_{\mathrm{i}}(i=1,2, \ldots, k)$ points are taken. We connect this points by $n$ - nonintersection $\operatorname{arcs} d_{1}, d_{2}, \ldots, d_{n}$ in such a way each $d_{t}$ corresponds to $A_{t}(t=1,2, \ldots, n)$ and $P_{i}$ and $P_{j}$ connected by $d_{t}$, if and only if $\alpha_{i}, \alpha_{j}$ have a common double point $A_{t}$ on their boundaries.Thus, a plane graph is obtained from regular projection of the knot $G$. From same consideration about class $\beta$, another graph $G^{d}$ is get. We call $G^{d}$ the dual graph of G. This method is called as Tait's method. Figure 2(a) and 2(b) show that graph and dual graph of Trefoil knot, respectively,

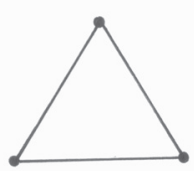

(a)

Figure 2

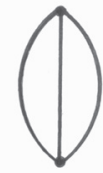

(b)

The regions of class $\alpha$ in all can be considered as a projection of a surface spanning $K$ which is twisted $180^{\circ}$ at each double point of projection. From this consideration, we define the sign of $d_{t}$ in such a way that it is + or - according as the surface is twisted at $A_{t}$ as the right screw or the left one, respectively (Figure 3 ).
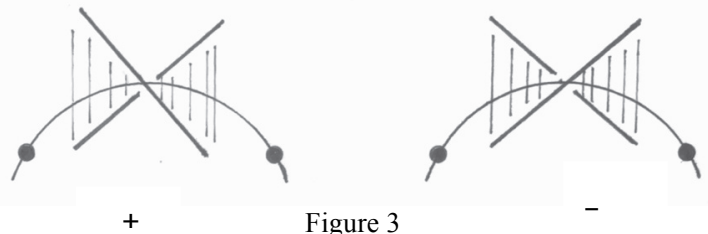

Figure 3

It is well known that every deformation of a knot is equivalent to a combination of the fundamental deformation (Reidemeister Movies) $\Omega_{1}, \Omega_{2}$ and $\Omega_{3}$ in [7].
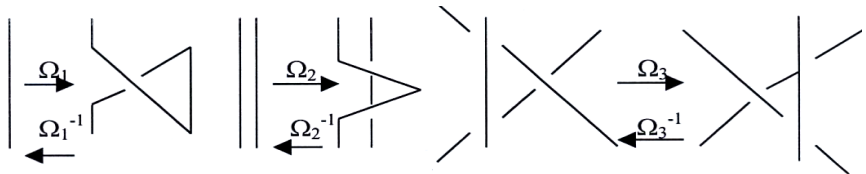

Figure 4

These fundamental deformations can be translated into the operations on graphs as follows [8].

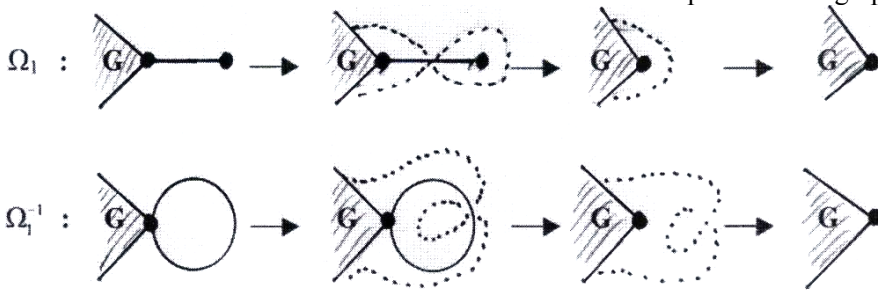




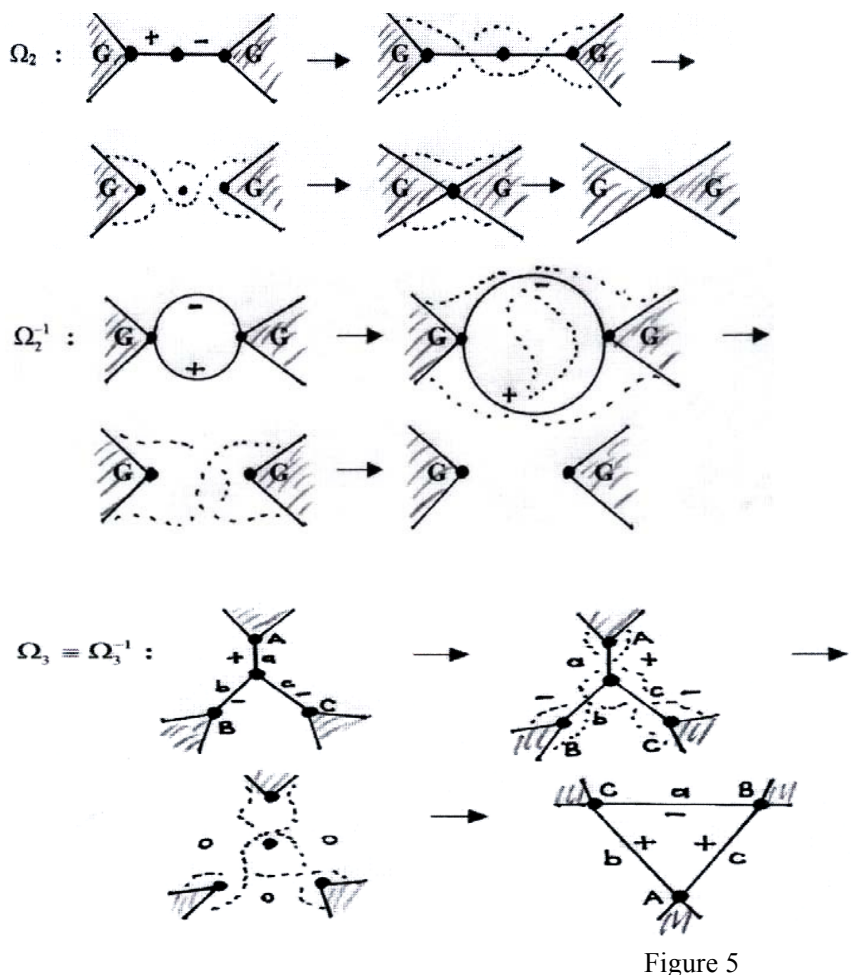

We recommend $[2,5,6]$ for details about this section.

\section{Knotdigraph Notation}

Firstly, signed graph is obtained from regular diagram of a knot by using Tait methods explained in Section 2.Secondly, orientation is given depending on the signed of the signed knot graph as Figure 6.If the signed of edge is +, orientation of this edge is given from a to $b$. If the signed of edge is -, orientation of this edge is given from $b$ to $a$. So we can get two directed graphs, when we consider graph and dual graph of a knot. We called this directed graph as knotdigraph.
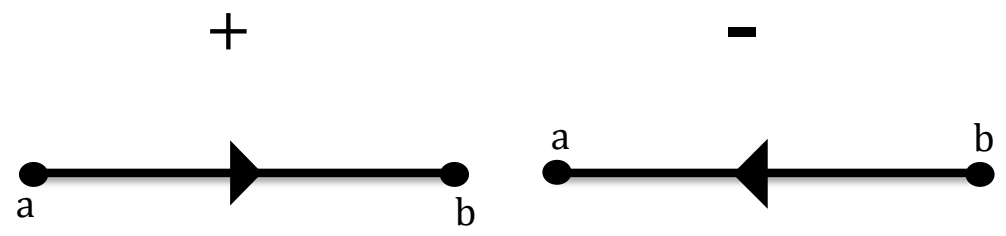

Figure 6. Orientation of knot graph depend on the signed of the signed knot graph

After that, every vertices of knot digraph are labelled by lowercases. Finally, the base is obtained from knot digraph by using quasi-pseudo metric for bitopology. Thus, every knot is matched with bitopology obtained by using this method.We called this method as Knotdigraph Notation

We can apply this method to dual graph of trefoil. Firstly we draw signed dula graph of oriented right-hand trefoil. Secondly, we draw knot digraph and label every vertices of knot digraph by 1,2 as a Figure 7

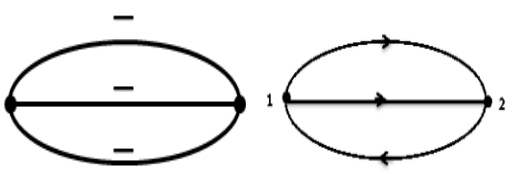

Figure 7 
After that we apply Reidemester deformation to labelled knot digraph as a Figure 8 . Adding point denoted by $\varepsilon_{1}^{12}$ depending on edge of knot

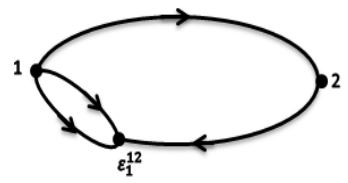

Figure 8

Finally, we obtained the base by using quasi-pseudo metrics mentioned in Section 3.Then, bitopology of dual graph of oriented right-hand trefoil is $\tau_{1}=\{\varnothing, X,\{1\},\{1,2\}\}, \tau_{2}=\left\{\varnothing, X,\left\{\varepsilon_{1}^{12}\right\},\left\{\varepsilon_{1}^{12}, 2\right\}\right\}$.

We get a bitopology by applying this method to knots $k_{m}$, where $n$ is crossing points of knot and $m=1,2$. We get some tables as follows.

\begin{tabular}{|c|c|}
\hline$k_{1}(k$ is odd $)$ & Bitopology associated with knot digraph \\
\hline $3_{1}$ & $\tau_{1}=\{\varnothing, X,\{1\},\{1,2\}\}, \tau_{2}=\left\{\varnothing, X,\left\{\varepsilon_{1}^{12}\right\},\left\{\varepsilon_{1}^{12}, 2\right\}\right\}$ \\
\hline $5_{1}$ & $\tau_{1}=\{\varnothing, X,\{1\},\{1,2\}\}, \tau_{2}=\left\{\varnothing, X,\left\{\varepsilon_{1}^{12}, \varepsilon_{2}^{12}\right\},\left\{\varepsilon_{1}^{12}, \varepsilon_{2}^{12}, 2\right\}\right\}$ \\
\hline $7_{1}$ & $\tau_{1}=\{\varnothing, X,\{1\},\{1,2\}\}, \tau_{2}=\left\{\varnothing, X,\left\{\varepsilon_{1}^{12}, \varepsilon_{2}^{12}, \varepsilon_{3}^{12}\right\},\left\{\varepsilon_{1}^{12}, \varepsilon_{2}^{12}, \varepsilon_{3}^{12}, 2\right\}\right\}$ \\
\hline $9_{1}$ & $\tau_{1}=\{\varnothing, X,\{1\},\{1,2\}\}, \tau_{2}=\left\{\varnothing, X,\left\{\varepsilon_{1}^{12}, \varepsilon_{2}^{12}, \varepsilon_{3}^{12}, \varepsilon_{4}^{12}\right\},\left\{\varepsilon_{1}^{12}, \varepsilon_{2}^{12}, \varepsilon_{3}^{12}, \varepsilon_{4}^{12}, 2\right\}\right\}$ \\
\hline$\vdots$ & $\vdots$ \\
\hline $\mathrm{k}_{1}$ & $\tau_{1}=\{\varnothing, X,\{1\},\{1,2\}\}, \tau_{2}=\left\{\varnothing, X,\left\{\varepsilon_{1}^{12}, \varepsilon_{2}^{12}, \ldots, \varepsilon_{\frac{k-1}{2}}^{12}\right\},\left\{\varepsilon_{1}^{12}, \varepsilon_{2}^{12}, \ldots, \varepsilon_{\frac{k-1}{2}}^{12}, 2\right\}\right\}$ \\
\hline
\end{tabular}

\begin{tabular}{|c|c|}
\hline$k_{1}(k$ is even) & Bitopology associated with knot digraph \\
\hline $44_{1}$ & $\tau_{1}=\{\varnothing, X,\{1\},\{1,2\},\{1,2,3\}\}, \tau_{2}=\left\{\varnothing, X,\left\{\varepsilon_{1}^{13}\right\},\left\{\varepsilon_{1}^{13}, 3\right\},\left\{\varepsilon_{1}^{13}, 2,3\right\}\right\}$ \\
\hline $6_{1}$ & $\begin{array}{c}\tau_{1}=\{\varnothing, X,\{1\},\{1,2\},\{1,2,3\},\{1,2,3,4\},\{1,2,3,4,5\}\} \\
\tau_{2}=\left\{\varnothing, X,\left\{\varepsilon_{1}^{15}\right\}\left\{\varepsilon_{1}^{15}, 5\right\}\left\{\varepsilon_{1}^{15}, 4,5\right\},\left\{\varepsilon_{1}^{15}, 3,4,5\right\},\left\{\varepsilon_{1}^{15}, 2,3,4,5\right\}\right\}\end{array}$ \\
\hline $8_{1}$ & $\begin{array}{c}\tau_{1}=\{\varnothing, X,\{1\},\{1,2\},\{1,2,3\},\{1,2,3,4\},\{1,2,3,4,5\},\} \\
\{1,2,3,4,5,6\},\{1,2,3,4,5,6,7\} \\
\tau_{2}=\left\{\begin{array}{c}\emptyset, X,\left\{\varepsilon_{1}^{17}\right\},\left\{\varepsilon_{1}^{17}, 7\right\},\left\{\varepsilon_{1}^{17}, 6,7\right\},\left\{\varepsilon_{1}^{17}, 5,6,7\right\}\left\{\varepsilon_{1}^{17}, 4,5,6,7\right\}, \\
\left\{\varepsilon_{1}^{17}, 3,4,5,6,7\right\},\left\{\varepsilon_{1}^{17}, 2,3,4,5,6,7\right\}\end{array}\right\}\end{array}$ \\
\hline $10_{1}$ & $\begin{array}{c}\tau_{1}=\left\{\begin{array}{c}\emptyset, X,\{1\},\{1,2\},\{1,2,3\},\{1,2,3,4\},\{1,2,3,4,5\},\{1,2,3,4,5,6\}, \\
\{1,2,3,4,5,6,7\},\{1,2,3,4,5,6,7,8\},\{1,2,3,4,5,6,7,8,9\}\end{array}\right\} \\
\tau_{2}=\left\{\begin{array}{c}\emptyset, X,\left\{\varepsilon_{1}^{19}\right\},\left\{\varepsilon_{1}^{19}, 9\right\},\left\{\varepsilon_{1}^{19}, 8,9\right\},\left\{\varepsilon_{1}^{19}, 7,8,9\right\}, \\
\left\{\varepsilon_{1}^{19}, 6,7,8,9\right\}\left\{\varepsilon_{1}^{19}, 5,6,7,8,9\right\}, \\
\left\{\varepsilon_{1}^{19}, 4,5,6,7,8,9\right\}\left\{\varepsilon_{1}^{19}, 3,4,5,6,7,8,9\right\},\left\{\varepsilon_{1}^{19}, 2,3,4,5,6,8,97\right\}\end{array}\right\}\end{array}$ \\
\hline$\vdots$ & $\vdots$ \\
\hline $\mathrm{k}_{1}$ & $\begin{array}{c}\tau_{1}=\{\varnothing, X,\{1\},\{1,2\}, \ldots,\{1,2, \ldots, \mathrm{k}-1\}\} \\
\tau_{2}=\left\{\begin{array}{c}\emptyset, X,\left\{\varepsilon_{1}^{1 k-1}\right\},\left\{\varepsilon_{1}^{1 k-1}, k-1\right\},\left\{\varepsilon_{1}^{1 k-1}, k-2, k-1\right\}, \\
\left\{\varepsilon_{1}^{1 k-1}, k-3, k-2, k-1\right\}, \ldots,\left\{\varepsilon_{1}^{1 k-1}, 2, \ldots, k-2, k-1\right\}\end{array}\right\}\end{array}$ \\
\hline$k_{2}(k$ is odd $)$ & Bitopology associated with knot digraph \\
\hline $5_{2}$ & $\begin{array}{c}\tau_{1}=\{\varnothing, X,\{1\},\{1,2\},\{1,2,3\},\{1,2,3,4\}\} \\
\tau_{2}=\left\{\varnothing, X,\left\{\varepsilon_{1}^{14}\right\},\left\{\varepsilon_{1}^{14}, 4\right\},\left\{\varepsilon_{1}^{14}, 3,4\right\},\left\{\varepsilon_{1}^{14}, 2,3,4\right\}\right\}\end{array}$ \\
\hline
\end{tabular}




\begin{tabular}{|c|c|}
\hline $7_{2}$ & $\tau_{1}=\{\varnothing, X,\{1\},\{1,2\},\{1,2,3\},\{1,2,3,4\},\{1,2,3,4,5\},\{1,2,3,4,5,6\}\}$ \\
$\tau_{2}=\left\{\begin{array}{c}\emptyset, X,\left\{\varepsilon_{1}^{16}\right\},\left\{\varepsilon_{1}^{16}, 6\right\},\left\{\varepsilon_{1}^{16}, 5,6\right\},\left\{\varepsilon_{1}^{16}, 4,5,6\right\}, \\
\left\{\varepsilon_{1}^{16}, 3,4,5,6\right\},\left\{\varepsilon_{1}^{16}, 2,3,4,5,6\right\}\end{array}\right\}$ \\
\hline $9_{2}$ & $\tau_{1}=\left\{\begin{array}{c}\emptyset, X,\{1\},\{1,2\},\{1,2,3\},\{1,2,3,4\},\{1,2,3,4,5\}, \\
\{1,2,3,4,5,6\},\{1,2,3,4,5,6,7\},\{1,2,3,4,5,6,7,8\}\end{array}\right\}$ \\
\hline$\vdots$ & $\tau_{2}=\left\{\begin{array}{c}\emptyset, X,\left\{\varepsilon_{1}^{18}\right\},\left\{\varepsilon_{1}^{18}, 8\right\},\left\{\varepsilon_{1}^{18}, 7,8\right\},\left\{\varepsilon_{1}^{18}, 6,7,8\right\},\left\{\varepsilon_{1}^{18}, 5,6,7,8\right\}, \\
\left\{\varepsilon_{1}^{18}, 4,5,6,7,8,\right\},\left\{\varepsilon_{1}^{18}, 3,4,5,6,7,8\right\},\left\{\varepsilon_{1}^{18}, 2,3,4,5,6,7,8\right\}\end{array}\right\}$ \\
\hline $\mathrm{k}_{2}$ & $\vdots$ \\
\hline & $\tau_{2}=\{\varnothing, X,\{1\},\{1,2\},\{1,2, \ldots, \mathrm{k}-1\}\}$ \\
$\left(\begin{array}{c}\emptyset, X,\left\{\varepsilon_{1}^{1 k-1}\right\},\left\{\varepsilon_{1}^{1 k-1}, k-1\right\},\left\{\varepsilon_{1}^{1 k-1}, k-2, k-1\right\}, \\
\left\{\varepsilon_{1}^{1 k-1}, k-3, k-2, k-1\right\}, \ldots,\left\{\varepsilon_{1}^{1 k-1}, 2, \ldots, k-2, k-1\right\}\end{array}\right\}$ \\
\hline
\end{tabular}

\begin{tabular}{|c|c|}
\hline$k_{2}(k$ is even $)$ & Bitopology associated with knot digraph \\
\hline $6_{2}$ & $\begin{array}{c}\tau_{1}=\{\varnothing, X,\{1\},\{1,2\},\{1,2,3\}\} \\
\tau_{2}=\left\{\varnothing, X,\left\{\varepsilon_{1}^{23}, \varepsilon_{1}^{13}\right\},\left\{\varepsilon_{1}^{23}, \varepsilon_{1}^{13}, 3\right\},\left\{\varepsilon_{1}^{23}, \varepsilon_{1}^{13}, 2,3\right\}\right\}\end{array}$ \\
\hline $88_{2}$ & $\begin{array}{c}\tau_{1}=\{\varnothing, X,\{1\},\{1,2\},\{1,2,3\}\} \\
\tau_{2}=\left\{\varnothing, X,\left\{\varepsilon_{1}^{23}, \varepsilon_{2}^{23}, \varepsilon_{1}^{13}\right\},\left\{\varepsilon_{1}^{23}, \varepsilon_{2}^{23}, \varepsilon_{1}^{13}, 3\right\},\left\{\varepsilon_{1}^{23}, \varepsilon_{2}^{23}, \varepsilon_{1}^{13}, 2,3\right\}\right\}\end{array}$ \\
\hline $10_{2}$ & $\begin{array}{c}\tau_{1}=\{\varnothing, X,\{1\},\{1,2\},\{1,2,3\}\} \\
\tau_{2}=\left\{\begin{array}{c}\emptyset, X,\left\{\varepsilon_{1}^{23}, \varepsilon_{2}^{23}, \varepsilon_{3}^{23}, \varepsilon_{1}^{13}\right\},\left\{\varepsilon_{1}^{23}, \varepsilon_{2}^{23}, \varepsilon_{3}^{23}, \varepsilon_{1}^{13}, 3\right\}, \\
\left\{\varepsilon_{1}^{23}, \varepsilon_{2}^{23}, \varepsilon_{3}^{23}, \varepsilon_{1}^{13}, 2,3\right\}\end{array}\right\}\end{array}$ \\
\hline$\vdots$ & $\vdots$ \\
\hline $\mathrm{k}_{2}$ & $\begin{array}{c}\tau_{1}=\{\varnothing, X,\{1\},\{1,2\},\{1,2,3\}\} \\
\tau_{2}=\left\{\begin{array}{c}\emptyset, X,\left\{\varepsilon_{1}^{23}, \varepsilon_{2}^{23}, \ldots, \varepsilon_{\frac{k-4}{2}}^{23}, \varepsilon_{1}^{13}\right\},\left\{\varepsilon_{1}^{23}, \varepsilon_{2}^{23}, \ldots, \varepsilon_{\frac{k-4}{2}}^{23}, \varepsilon_{1}^{13}, 3\right\}, \\
\left\{\varepsilon_{1}^{23}, \varepsilon_{2}^{23}, \ldots, \varepsilon_{\frac{k-4}{2}}^{23}, \varepsilon_{1}^{13}, 2,3\right\}\end{array}\right\}\end{array}$ \\
\hline
\end{tabular}

\section{References}

1. Alexander, J.W., G.B., On types of knotted curve. Ann. Math., 28, 562-586, 1956.

2. Bankwitz, C., Uber die Torsionszahlen der alternierden Knoten. Math. Ann., 103, 145-161, 1930.

3. Girija B., Pilakkat R., Bitopological spaces associated with digraphs, South Asian Journal of Mathematics, Vol.3 (1):56-65, 2013.

4. Kelley J.C., Bitopological Spaces, Proc. London Math.(3), 13, 71-89, 1963.

5. Murasugi, K., On invariants of graphs with applications to knot theory. Trans. Amer. Math. Soc., 314, 1-49, 1989.

6. Murasugi, K., Knot Theory and Its Application.Boston,1993.

7. K. Reidemeister: Knotentheorie, Julius Springer, 1932.

8. Yajima T., Kinoshita S., On the graphs of knots, Osaka Math. J., 9, 155-163, 1957. 